\title{
INTERVENÇÕES COGNITIVAS EM IDOSOS COM DOENĢA DE ALZHEIMER: UMA REVISÃO INTEGRATIVA DA ATUAÇÃO DA TERAPIA OCUPACIONAL
}

\section{Lilian Dias Bernardo ${ }^{1}$}

resumo

Introdução: Idosos com Doença de Alzheimer, conforme a evolução da doença, apresentam alterações cognitivas e prejuízo funcional. Objetivo: Identificar e analisar as técnicas e benefícios da intervenção cognitiva utilizadas por terapeutas ocupacionais no tratamento de idosos com Alzheimer. Métodos: Foi realizada revisão integrativa da literatura, no período de 10 anos (2006 a 2016), nos idiomas inglês, português e espanhol. Optou-se pela busca em oito fontes de informação. Resultados: Foram identificados nove artigos que atenderam aos critérios de seleção, dos quais somente um estudo nacional foi encontrado. Nas intervenções, a terapia de reminiscência, de orientação para a realidade e o treino cognitivo são as técnicas mais utilizadas para melhorar um domínio cognitivo específico e há evidências que atestam benefícios na participação do idoso em ocupações. Recomenda-se maior número de ensaios clínicos para

1 Graduada em Terapia Ocupacional. Doutora em Saúde Coletiva na área de Políticas, Planejamento e Administração em Saúde. Docente do Curso de Graduação em Terapia Ocupacional do Instituto Federal de Educação, Ciência e Tecnologia do Rio de Janeiro (IFRJ). E-mail: lilian.bernardo@ifrj.edu.br. 
aumentar a confiabilidade dos resultados encontrados nesta revisão. Verifica-se a necessidade de investigar os benefícios dessas técnicas em idosos em estágio mais avançado da doença. Conclusão: A evidência disponível aponta que a intervenção cognitiva parece manter ou resultar em melhora dos déficits cognitivos, mas ainda pouco se sabe sobre a perpetuação e generalização dos efeitos dessas técnicas em idosos com Alzheimer.

palavras-chave

Terapia ocupacional. Doença de Alzheimer. Idoso. Cognição. Produção Científica e Tecnológica.

\section{Introdução}

Em face da elevada prevalência mundial e do impacto social desencadeado pela Doença de Alzheimer (DA), a Organização Mundial de Saúde passou a considerar, desde 2012, essa doença e as outras demências correlatas entre as prioridades da saúde pública (DUTHEY, 2013; WHO, 2012). Essa condição de saúde acomete, com maior prevalência, os idosos. Apresenta, de forma gradual e irreversível, alterações nos múltiplos domínios cognitivos e/ou sintomas neuropsiquiátricos e comportamentais (ALZHEIMER'S DISEASE INTERNATIONAL, 2014), com impactos no funcionamento cognitivo, no engajamento em ocupações significativas e nas interações sociais (ALZHEIMER'S ASSOCIATION, 2013).

Para identificação dessas pessoas com Alzheimer, foi estabelecido um consenso nacional sobre os critérios diagnósticos. Estes se baseiam na análise de exames complementares e nos relatos de velocidade da progressão da doença, piora cognitiva e queixas iniciais/proeminentes de déficits cognitivos associados à memória, linguagem, função executiva e visuo-espacial (FROTA et al., 2011). Ademais, constituem como ferramentas opcionais para diagnósticos, a utilização do Manual Diagnóstico e Estatístico de Transtornos Mentais da Associação Americana de Psiquiatria (DSM - V), que descreve a doença dentro da categoria dos Transtornos Neurocognitivos Leves e Maiores devido à Doença de Alzheimer (APA, 2014) e os critérios da Classificação Internacional de Doenças e problemas relacionados à saúde (CID-10) da Organização Mundial de Saúde (OMS, 1993). 
Considerando o impacto da doença na funcionalidade e no intuito de contribuir para o aumento das habilidades cognitivas dos idosos com Alzheimer e consequente diminuição da assistência prestada pelo cuidador, a literatura na área da Terapia Ocupacional (TO) elenca as intervenções cognitivas entre as ações com efeitos benéficos na funcionalidade dos demenciados (THINNES; PADILLA, 2011). De acordo com Loureiro et al. (2011), as metas interventivas adotadas com essa população podem almejar a: (1) remediação: quando se avaliam os domínios cognitivos alterados e o foco é melhorar diretamente a função cognitiva que se encontra prejudicada, no intuito de que esses ganhos se traduzam em melhorias nas atividades; (2) compensação: ao trabalhar as habilidades que estão preservadas e mecanismos alternativos, uma vez que a função alterada não pode ser recuperada; e/ou a (3) substituição: pelo ensino de diferentes estratégias que ajudem o sujeito a minimizar os problemas resultantes das disfunções cognitivas. As abordagens não são excludentes e, normalmente nos tratamentos, elas são usadas de forma combinada.

Na intervenção cognitiva, várias técnicas podem ser empregadas: comportamentais, de orientação para a realidade, terapia de reminiscências e técnicas de validação (RADOMSKI; DAVIS, 2013), bem como o treino repetitivo (SOARES, V.; SOARES, C.; CAIXETA, 2012). No entanto, ainda são incipientes as evidências que abordam os efeitos dessas técnicas em idosos com ou sem déficits cognitivos.

Nesse panorama, o objetivo do artigo é analisar os efeitos das intervenções cognitivas em idosos com doença de Alzheimer durante o processo terapêutico ocupacional. Assim, a questão norteadora para a pesquisa foi: Qual o impacto das intervenções cognitivas na funcionalidade dos idosos com doença de Alzheimer?

\section{Materiais e Métodos}

Trata-se de uma revisão de literatura do tipo integrativa que evidencia o uso de intervenções cognitivas realizadas por terapeutas ocupacionais junto a idosos com doença de Alzheimer. O recorte temporal foi de 10 anos (meses de janeiro de 2006 a 2016) e as buscas foram realizadas em fevereiro e março de 2016. As fontes de informação selecionadas foram: Scopus, Web of Science, MEDLINE/PubMed (via National Library of Medicine), Cumulative Index to Nursing and Allied Health Literature (CINAHL), PsycINFO ${ }^{\circledR}$, Literatura Latino-Americana e do Caribe em Ciências da Saúde (LILACS), Scientific Eletronic Library on Line (SciELO), Occupational Therapy Systematic Evaluation of Evidence 
(OTseeker) e Physiotherapy Evidence Database (PEDro). A escolha dessas fontes se deu por serem de maior prestígio científico na área multidisciplinar, com cobertura internacional ou da América Latina e Caribe, bem como bases de dados específicas da Terapia Ocupacional ou áreas afins.

Independente do livre acesso às publicações, como critérios de inclusão foram considerados os artigos que abordam: a) a doença de Alzheimer em idosos, em qualquer fase da doença, sem restringir quanto à adesão ao tratamento; b) a participação de terapeuta ocupacional; c) enfoque nas intervenções cognitiva; d) nos idiomas português, inglês e espanhol. Como critérios de exclusão, foram descartadas: a) publicações que abordavam intervenções sem o enfoque na cognição; b) revisões de literatura; c) documentos que abordavam os outros tipos de demência; e, d) resumos de congressos, anais e notas prévias.

Para definição dos termos de busca, foi feita consulta no Descritores em Ciências da Saúde e foram estipulados: "comportamento", "meio ambiente" e "cognição", que foram combinados com "Doença de Alzheimer" e "Terapia Ocupacional" e suas expressões na língua inglesa e em espanhol. Os termos "ocupação" e "atividades" também foram usados na busca. Foram utilizados os operadores booleanos AND e OR para combinação. As estratégias construídas, as expressões de busca e os resultados são apresentadas na Tabela 1.

Tabela 1 - Fontes de informação, expressões de busca e resultados dos documentos identificados.

\begin{tabular}{|c|c|c|}
\hline $\begin{array}{l}\text { Fontes de } \\
\text { Informação }\end{array}$ & Expressões de Busca & Resultado \\
\hline $\begin{array}{l}\text { CINAHL with Full } \\
\text { Text (EBSCO) }\end{array}$ & $\begin{array}{l}\text { ("occupational therapy" OR "Occupational therapy/ } \\
\text { methods") AND ("Alzheimer" OR "Alzheimer disease" OR } \\
\text { "Alzheimer's disease")AND ("behavior" OR "environment" OR } \\
\text { "cognition" OR "occupation" OR "activities") }\end{array}$ & 59 \\
\hline LILACS & $\begin{array}{l}\text { "Alzheimer" [Palavras] and "occupational therapy" [Palavras] } \\
\text { OR "Behavior" [Palavras] and "Alzheimer disease" [Pala- } \\
\text { vras] OR "environment" [Palavras] and "Alzheimer disease" } \\
\text { [Palavras] }\end{array}$ & 77 \\
\hline $\begin{array}{l}\text { MEDLINE/ } \\
\text { PubMed (via } \\
\text { National Library of } \\
\text { Medicine) }\end{array}$ & $\begin{array}{l}\text { (("occupational therapy"[All Fields] OR "Occupational the- } \\
\text { rapy/methods"[All Fields]) AND ("Alzheimer"[All Fields] OR } \\
\text { "Alzheimer disease"[All Fields] OR "Alzheimer's disease"[All } \\
\text { Fields])) AND ("behavior"[All Fields] OR "environment"[All } \\
\text { Fields] OR "cognition"[All Fields] OR "occupation"[All Fields] } \\
\text { OR "activities"[All Fields]) }\end{array}$ & 120 \\
\hline
\end{tabular}




\begin{tabular}{|c|c|c|}
\hline OTseeker & $\begin{array}{l}\text { ("occupational therapy" OR "Occupational therapy/ } \\
\text { methods") AND ("Alzheimer" OR "Alzheimer disease" OR } \\
\text { "Alzheimer's disease") AND ("behavior" OR "environment" } \\
\text { OR "cognition" OR "occupation" OR "activities") }\end{array}$ & 09 \\
\hline PsycINFO & $\begin{array}{l}\text { Any Field: "occupational therapy" OR "Occupational therapy/ } \\
\text { methods" AND Any Field: "Alzheimer" OR "Alzheimer } \\
\text { disease" OR "Alzheimer's disease" AND Any Field: "beha- } \\
\text { vior" OR "environment" OR "cognition" OR "occupation" OR } \\
\text { "activities" }\end{array}$ & 157 \\
\hline PEDro & "Alzheimer" OR "dementia" & 31 \\
\hline $\begin{array}{l}\text { Scielo Citation } \\
\text { Index }\end{array}$ & $\begin{array}{l}\text { Tópico: (occupational therapy) AND Tópico: (Alzheimer } \\
\text { disease) OR Tópico: (behavior) ANDTópico: (Alzheimer } \\
\text { disease) OR Tópico: (environment) AND Tópico: (Alzheimer } \\
\text { disease) }\end{array}$ & 43 \\
\hline Scopus & $\begin{array}{l}\text { TITLE-ABS-KEY ("Occupational Therapy" OR "Occupatio- } \\
\text { nal therapy/methods") AND TITLE-ABS-KEY ("behavior" } \\
\text { OR "environment" OR "cognition" OR "occupation" OR } \\
\text { "activities") AND TITLE-ABS-KEY ( "Alzheimer" OR "Alzheimer } \\
\text { disease" OR "Alzheimer's disease" ) }\end{array}$ & 158 \\
\hline Web of Science & $\begin{array}{l}\text { Tópico: ("occupational therapy" OR "Occupational therapy/ } \\
\text { methods") AND Tópico: ("Alzheimer" OR "Alzheimer disease" } \\
\text { OR "Alzheimer's disease") AND Tópico: ("behavior" OR "envi- } \\
\text { ronment" OR "cognition" OR "occupation" OR "activities") }\end{array}$ & 41 \\
\hline $\begin{array}{c}\text { Total de busca de } \\
\text { artigos }\end{array}$ & & 695 \\
\hline
\end{tabular}

Fonte: Tabela elaborada pela autora, 2016.

Os documentos encontrados foram exportados o software EndNote ${ }^{\circledR}$ Web para armazenamento e organização do processo de seleção do corpus da pesquisa (Figura 1).

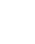




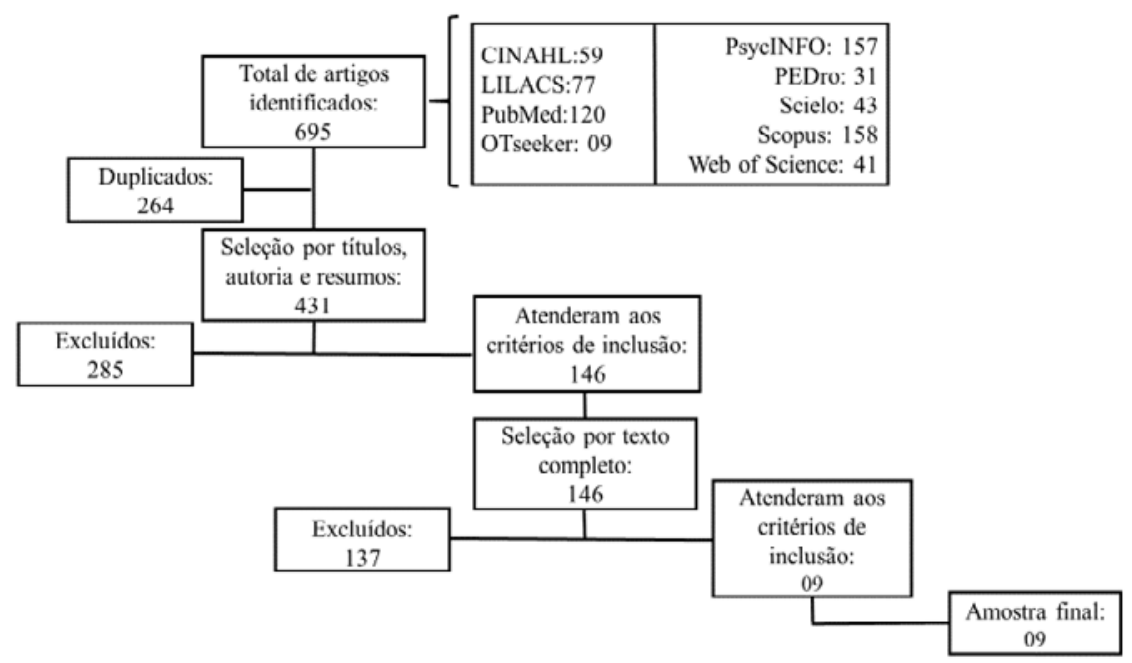

Fonte: Figura elaborada pela autora, 2016.

Na primeira seleção, o número de documentos identificados que atendiam aos critérios de inclusão foi de 146. Após a leitura na íntegra dos artigos selecionados, 79 artigos abordavam o processo de Terapia Ocupacional em idosos com Alzheimer. Dos 67 artigos excluídos, 28 artigos foram eliminados pela metodologia utilizada, em que foram utilizados estudos de revisão de literatura ou textos teóricos; 34 trabalhos não focavam no idoso com DA e/ou na atuação da Terapia Ocupacional; 05 artigos apresentavam o resumo em inglês, mas os idiomas do texto completo não atendiam aos critérios de inclusão.

Das 79 publicações relacionadas às inúmeras possibilidades de avaliação e intervenção terapêutica ocupacional (sobre modificação ambiental, educação em saúde, cuidado ao cuidador, treino de ocupações, entre outros), somente nove artigos abordavam o tema investigado, que constituiu o corpus desta pesquisa. Posteriormente, para análise e sistematização dos dados obtidos, foi construído um formulário para organização dos resultados, submetidos, então, a um processo de categorização temática. 
Para caracterizar o corpus da pesquisa, observa-se que a maioria das publicações (89\%) foi feita nos últimos anos (2010 a 2016). Seis artigos possuíam o terapeuta ocupacional (TO) na autoria principal e somente uma publicação nacional foi identificada ( $n^{\circ}$ 2). A análise das publicações é apresentada na Tabela 2. Entre as intervenções cognitivas, foram encontradas nesse estudo: a estimulação cognitiva, o treino cognitivo e a reabilitação cognitiva. 
0
0
0
$\square$
$\square$
$\square$

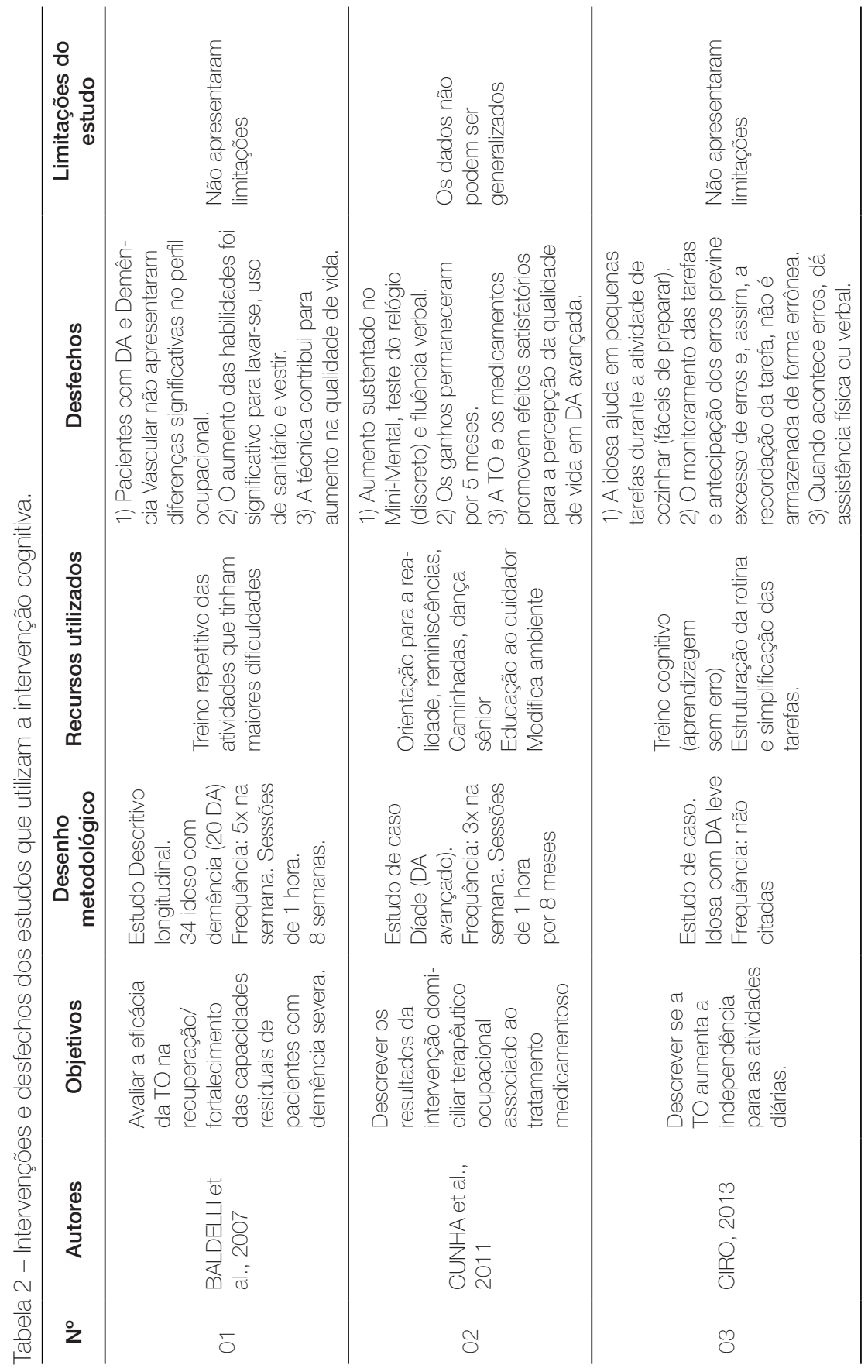




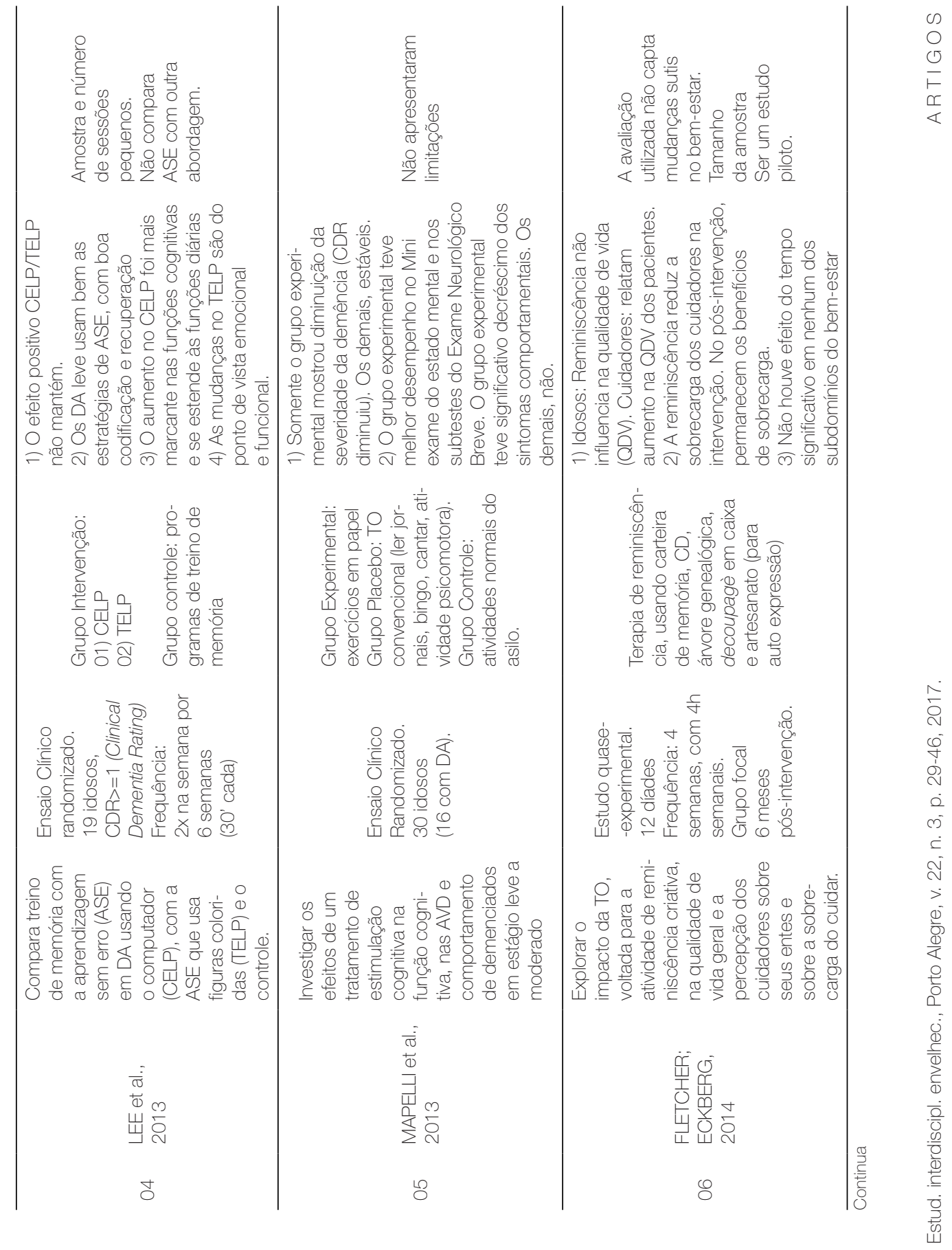


0
0
0
$\square$
$\square$
$\square$

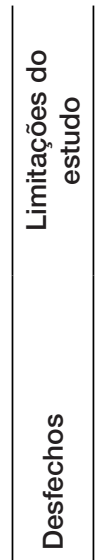

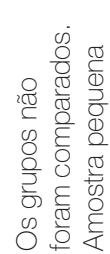

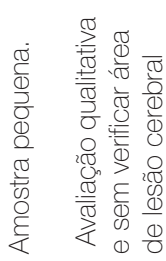

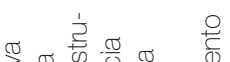

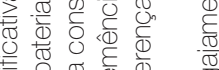

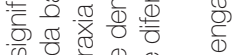

क.

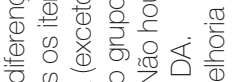

(1) 은

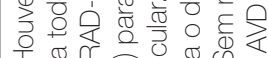

I

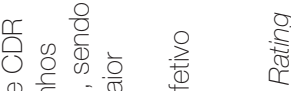

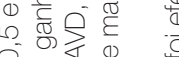

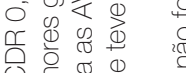

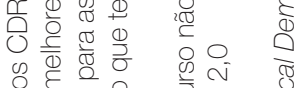

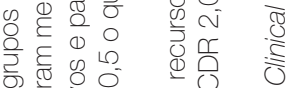

心

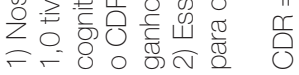

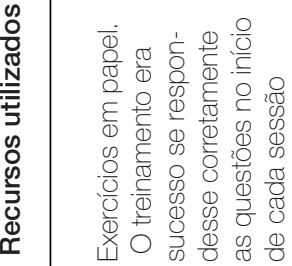

ำ

o

ก่

인

ल

$\dot{\varepsilon}$

กิ

$\frac{10}{8}$

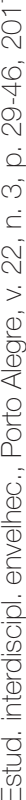

윙

एँ

$\frac{\frac{c}{D}}{d}$

$\frac{\bar{\alpha}}{\mathrm{O}}$

恶

ํำ

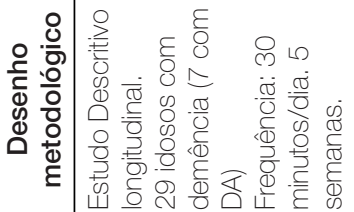

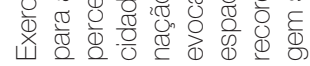

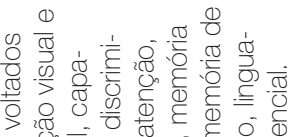

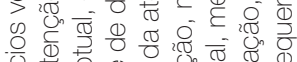

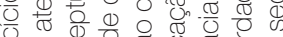

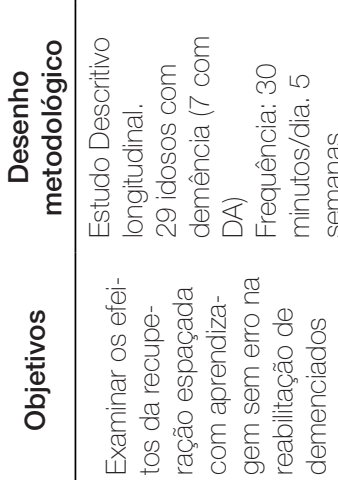

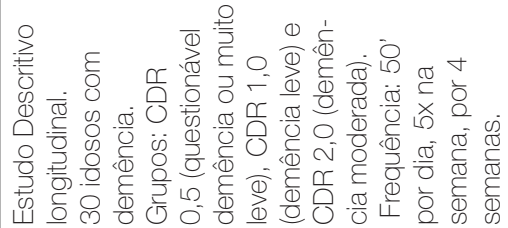

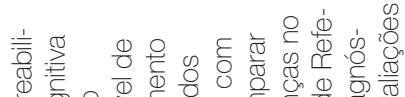

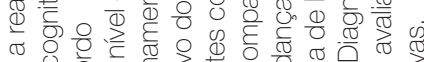

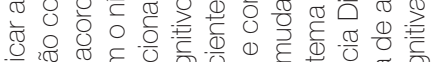

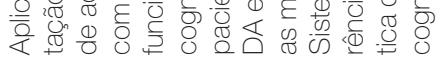

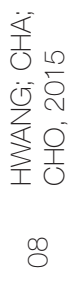

$\infty$ 


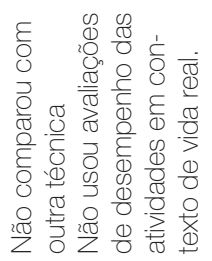

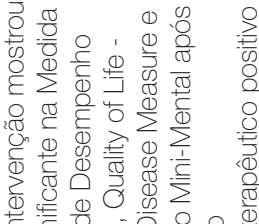

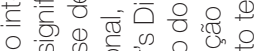

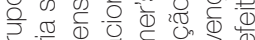

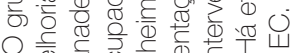

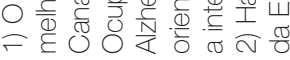

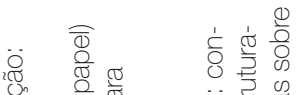

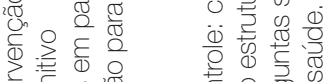

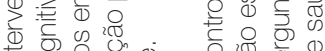

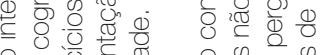

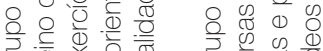

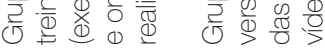

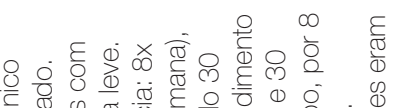

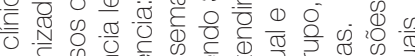

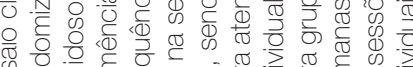
क

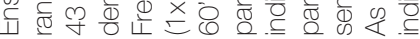

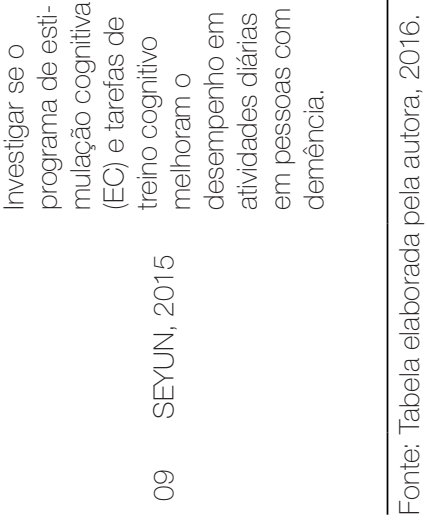


Nessa modalidade de intervenção, a terapia de reminiscência (TR) foi a técnica utilizada por Cunha et al. (2011) (no 02) e Fletcher e Eckberg (2014) (nº 06). É uma estratégia que utiliza materiais antigos para estimular memórias e permitir que o idoso compartilhe vivências e valores (CHAVES; PRADO; CAIXETA, 2012), reflexões sobre um passado significativo (SERRANI AZCURRA, 2012), expressões de sentimentos e busca ou incentivo para resolução de conflitos, se existentes (CHAVES; PRADO; CAIXETA, 2012). A literatura indica que, em grupos, contribui para o aumento da participação social e bem-estar entre os idosos com demências (LETTS et al., 2011)

No estudo $n^{0} 02$, a reminiscência foi associada à modificação ambiental e à terapia de orientação para a realidade a fim de melhorar a funcionalidade do idoso (ASSIS, M.; ASSIS, L.; CARDOSO, 2013). Os desfechos para o idoso em DA avançada estavam relacionados a ganho na fluência verbal, discreta melhora na função executiva (identificada pelo teste do relógio) e, de forma inesperada, aumento sustentado nos escores do Mini-Exame do Estado Mental - MEEM (contrário ao que é apresentado na literatura), principalmente nos itens de orientação temporal e espacial. O cuidador associou o programa à diminuição de sua sobrecarga de trabalho.

Já no estudo $n^{\circ} 06$, a abordagem não apresentou efeito na atenção, afeto, interesse e autoestima dos idosos (FLETCHER; ECKBERG, 2014). Os idosos não relacionaram a terapia de reminiscências à melhoria na qualidade de vida. Em contraponto, os cuidadores associaram ao aumento da qualidade de vida e diminuição da sobrecarga. Esses resultados diferem da revisão sistemática de Yen e Lin (2018) que atribui a essa técnica melhorias nos domínios cognitivos, autoestima e satisfação pessoal, assim como redução dos sintomas depressivos e ansiedade. De forma semelhante, o ensaio clínico randomizado de Serrani Azcurra (2012) diverge do estudo revisado, pois os idosos associaram a reminiscência à melhor qualidade de vida e maior engajamento em ocupações.

A terapia de orientação para realidade (TOR) foi a técnica empregada no estudo de Seyun (2015) ( $n^{\circ}$ 09), com melhorias para orientação no tempo e espaço, retratados nos valores finais do Mini Exame do Estado Mental. Essa técnica foi associada ao treino cognitivo, estratégias compensatórias e gerenciamento de estresse. $O$ grupo intervenção também apresentou melhoria na qualidade de vida, bem como aumento de satisfação no desempenho de ocupações significativas. Apesar de não serem relatados nessa revisão, a literatura aponta a terapia de reminiscência e a de orientação para a realidade como abordagens 
que podem levar a discreto benefício no humor de pacientes com Alzheimer (CHAVES; PRADO; CAIXETA , 2012).

Apesar da pesquisa de Cunha et al. (2011) apresentar benefícios em idosos na fase severa da doença, trata-se de um estudo de caso e os dados não são generalizáveis. Além disso, Cunha et al. (2011) e Seyun (2015) utilizaram diferentes técnicas em suas intervenções e não se sabe o efeito delas em separado. A mesma cautela deve ser direcionada ao artigo $n^{\circ} 06$, por ser um estudo piloto, com amostra pequena e que usaram instrumentos de avaliação que não são capazes de captar mudanças sutis no bem-estar (FLETCHER; ECKBERG, 2014). As limitações estão em consonância com a revisão sistemática de Wood, Womack e Hooper (2009) para análise da terapia de reminiscência, que apontam para falhas no tamanho amostral, nas metodologias e com resultados não significativos estatisticamente, necessitando de estudos mais robustos e de melhor qualidade.

\section{Reabilitação Cognitiva}

O treino repetitivo foi o tipo de reabilitação utilizado por Baldelli et al. (2007) (nº 01) para o desempenho de atividades em um contexto do mundo real. O treino sistemático para realização das atividades de vestir, banho e uso do vaso sanitário associou-se ao maior engajamento nessas ocupações do cotidiano dos participantes. Segundo Sohlberg e Mateer (2010), "o treinamento repetitivo ativa, mais consistentemente, os mesmos grupos de neurônios da rede danificada, permitindo uma reconectividade mais rápida e eficiente", que facilita a recuperação dos mecanismos biológicos e reorganização da função do cérebro.

\section{Treino Cognitivo}

Os artigos no 13, 04, 05, 07 e 08 adotaram o treino cognitivo e, em sua maioria, utilizaram os exercícios em papel como recurso terapêutico. Nesse treino, várias estratégias podem ser empregadas para que o paciente possa utilizar melhor a informação cognitiva: aprendizagem sem erro (ASE), pistas eliminadas, pareamento de estímulos e recuperação espaçada (RADOMSKI; DAVIS, 2013). O estudo $n^{0} 03$, o treino cognitivo com uso da aprendizagem sem erro foi associado a maior engajamento em ocupações (preparo de alimentos). No entanto, trata-se de um estudo de caso. O mesmo foi encontrado nos estudos de Hwang, Cha e Cho (2015) (n⿳0 08) para idosos na fase inicial da doença. 
O estudo de Lee et al. (2013) (n⿳0 04) utilizou a ASE em atividades no computador e em papel; e, o estudo de Jang, Lee e Yoo (2015) (nº 07) examinou exercícios com questões, com a abordagem da ASE e da recuperação espaçada. No estudo $n^{0}$ 04, os idosos apresentaram boa codificação e recuperação de informações. Ao grupo do computador, houve melhor resposta para as funções cognitivas, pois o feedback audiovisual do computador parece ser uma melhor estratégia de aprendizagem. Para os que utilizaram as atividades em papel, as mudanças são do ponto de vista emocional e funcional, que pode ser atribuído ao suporte direto dado pelo terapeuta ocupacional. Por outro lado, no estudo de Jang, Lee e Yoo (2015) (nº 07), as abordagens não apresentaram nenhuma diferença nos domínios cognitivos nem para o engajamento em atividades diárias.

As limitações dos estudos no 04 e 07 se referem ao tamanho amostral, metodologia e ausência de comparação entre técnicas. A literatura também aponta para o efeito limitado de perpetuação dos benefícios (SCHABER; LIEBERMAN, 2010). Werd et al. (2013) e Wu et al. (2014) afirmam que as técnicas de ASE e recuperação espaçada, respectivamente, são efetivas somente para conhecimentos relevantes para o idoso com DA. Acresce a isso, relatos sobre a ineficiência dessas técnicas em transferir os ganhos cognitivos para o desempenho nas atividades do cotidiano (SOARES, V.; SOARES, C.; CAIXETA, 2012). Apesar de compreender que todas as investigações possuem barreiras e facilitadores para a condução de seus planos de trabalho, alguns artigos desta revisão não explicitaram quais foram as limitações encontradas (estudos $\mathrm{n}^{\mathrm{o}}$ 01, 03 e 05).

Por sua vez, o estudo de Mapelli et al. (2013) (n⿳0 05) ressalta que o treino cognitivo apresentou melhoria significativa dos sintomas cognitivos, com redução da severidade da demência e influência na regulação do comportamento. Em consonância com os artigos revisados, a revisão sistemática de Woods et al. (2012) avaliou a eficácia e o impacto da estimulação cognitiva em dementados. Na análise, o treino cognitivo aumentou a cognição em DA inicial e intermediária, com resultados superiores à terapia medicamentosa. No entanto, os estudos tinham qualidade variável e não se sabe os benefícios em longo prazo.

Na mesma direção, o treino cognitivo em pacientes com demência inicial e moderada foi considerado apropriado para preservar a função cognitiva e promover níveis melhores de engajamento em ocupações significativas (YUILL; HOLLIS, 2011). À semelhança, a revisão sistemática de Bahar-Fuchs, Clare e Woods (2013) relata benefícios do treino cognitivo no engajamento em atividades diárias em idosos com DA inicial, mas isso não se aplica ao treino repetitivo. 
Mesmo com os benefícios divulgados nas pesquisas, é necessário cautela ao se adotar as intervenções cognitivas, pois pouco se sabe dos benefícios após o término da intervenção. Há necessidade de estudos com follow-up para avaliar o tempo em que os ganhos permanecem no paciente, mesmo sabendo do caráter progressivo da doença. Acresce a isso, reflexões sobre a frequência e tempo das sessões. Não há um tempo padronizado entre as pesquisas para que se possa concluir a respeito do período mínimo necessário para estimular e obter ganhos cognitivos e funcionais.

Ademais, somente dois estudos foram realizados com idosos na fase avançada da doença (no 01, 02). Sabe-se que nesse estágio, as funções cognitivas estão seriamente deterioradas e criam barreiras para o desempenho ocupacional. Isso pode justificar a escassez de estudos nessa fase da doença. Em contrapartida, outros autores relatam que, na fase severa, os idosos ainda podem manter algumas habilidades comunicativas e sociais, mostrando alguma capacidade de readaptação funcional (MANSUR et al., 2005). Dessa forma, são necessários mais estudos com os idosos em estágio avançado para que se possa avaliar se existem técnicas de estimulação cognitiva que possam trazer benefícios, mesmo que pequenos, ao funcionamento cognitivo e social.

\section{Considerações Finais}

A doença de Alzheimer modifica profundamente as interações sociais dos idosos e suas relações com o mundo. Nesse sentido, é um desafio para os profissionais de saúde - aqui foi analisado o papel do terapeuta ocupacional - considerar os aspectos pessoais, sociais, econômicos ou ambientais que condicionam o processo de saúde e doença para que se possa planejar intervenções que buscam pela dignidade e qualidade de vida.

Dentre as inúmeras possibilidades de intervenção do terapeuta ocupacional, as evidências científicas atuais apontam que as intervenções cognitivas apresentam benefícios para uma específica função cognitiva e até para melhor desempenho em atividades do cotidiano, dependendo da técnica adotada. No entanto, há necessidade de estudos para verificar a perpetuação dos efeitos terapêuticos, bem como os reais benefícios nos idosos em estágios mais avançados da doença. 


\title{
abstract
}

Introduction: Older adults with Alzheimer's disease, according to the evolution of the disease, present cognitive alterations and functional impairment. Objective: Identify and analyse the techniques and benefits of cognitive intervention used by occupational therapists in the treatment of older adults with Alzheimer's disease. Methods: An integrative review of literature was carried out, over a period of 10 years (from 2006 to 2016), in English, Portuguese and Spanish. We chose to search 8 databases which were used as sources of information. Results: Nine articles that met the selection criteria were identified, of which only one national study was found. In interventions, reminiscence therapy, orientation to reality and cognitive training are the most used to gain a specific cognitive domain, as well as benefits in the older adult's participation in occupations. More clinical trials are recommended to increase the reliability of the results found in this review. There is a need to investigate the benefits of these techniques in the older adults at an advanced stage of the disease. Conclusion: Available evidence suggests that cognitive intervention may maintain or result in improvement of cognitive deficits; however, there is a need for more robust studies about the perpetuation and generalization of the effects of these techniques in elderly people with Alzheimer's disease.

keywords

Occupational Therapy. Alzheimer Disease. Aged. Cognition. Scientific and Technical Activities.

referências

\begin{abstract}
ALZHEIMER'S ASSOCIATION. 2013 Alzheimer's disease fact and figures. Alzheimer's \& Dementia, Chicago, v. 9, n. 2, 2013. Disponível em: <https://www.alz.org/downloads/ facts_figures_2013.pdf >. Acesso em: 10 abr. 2018.
\end{abstract}

ALZHEIMER'S DISEASE INTERNATIONAL. World Alzheimer Report 2014: dementia and risk reduction - an analysis of protective and modifiable factors. Londres: ADI, 2014. 
AMERICAN PSYCHIATRIC ASSOCIATION (APA). Manual Diagnóstico e Estatístico de Transtornos Mentais (DSM V). Tradução de Maria Inês Corrêa Nascimento. Revisão técnica de Aristides Volpato Cordioli. 5. ed. Porto Alegre: Artmed, 2014.

ASSIS, Marcela Guimarães; ASSIS, Luciana de Oliveira; CARDOSO, Adnaldo Paulo. Reabilitação das atividades diárias. In: MALLOY-DINIZ, Leandro Fernandes; FUENTES, Daniel; COSENZA, Ramon Moreira. (Org.) Neuropsicologia do envelhecimento: uma abordagem multidimensional. Porto Alegre: Artmed, 2013. p. 360-377.

BAHAR-FUCHS, Alex; CLARE, Linda; WOODS, Bob. Cognitive training and cognitive rehabilitation for mild to moderate Alzheimer's disease and vascular dementia. Cochrane Database Systematic Reviews, San Diego, v. 5, n. 6, CD003260, 2013

BALDELLI, Maria Valéria et al. Occupational therapy and dementia: the experience of an Alzheimer special care unit. Archives of Gerontology and Geriatrics, Amsterdã, v. 44, n. 1, p. 49-54, 2007. Suplemento.

CHAVES, Moysés; PRADO, Carla; CAIXETA, Leonardo. Tratamento dos sintomas psicológicos e comportamentais da doença de Alzheimer. In: CAIXETA, Leonardo et al. Doença de Alzheimer. Porto Alegre: Artmed, 2012. p. 407-416.

$\mathrm{CIRO}$, Carrie. Second nature: Improving occupational performance in people with dementia through role-based, task-specific training. Occupational Therapy Practice, Bethesda, v. 18, n. 3, p. 9-12, jan. 2013

CUNHA, Fabiana Carla Matos da et al. Abordagem funcional e centrada no cliente na reabilitação de idoso com demência de Alzheimer: relato de caso. Revista de Terapia Ocupacional da Universidade de São Paulo, São Paulo, v. 22, n. 2, p. 145-152, maio/ ago. 2011.

DUTHEY, Béatrice. Background Paper 6.11: Alzheimer Disease and other Dementias. Genebra: OMS, 2013.

FLETCHER, Tina; ECKBERG, Johnathan. The effects of creative reminiscing on individuals with dementia and their caregivers: a pilot study. Physical \& Occupational Therapy in Geriatrics, Ontário, v. 32, n. 1, p. 68-84, 2014.

FROTA, Norberto A. F. et al. Critérios para o diagnóstico de doença de Alzheimer. Dementia \& neuropsychologia, São Paulo, v. 5, p. 5-10, jun. 2011. Suplemento.

HWANG, Jung-Ha; CHA, Hyun-Gyu.; CHO, Hyuk-Shin. The effects of cognitive rehabilitation in Alzheimer's dementia patients' cognitive assessment reference diagnosis system performance based level of cognitive functioning. Journal of Physical Therapy Science, Tóquio, v. 27, n. 9, p. 2875-2877, 2015.

JANG, Jong Sik.; LEE, Jae Shin; YOO, Doo Han. Effects of spaced retrieval training with errorless learning in the rehabilitation of patients with dementia. Journal of Physical Therapy Science, Tóquio, v. 27, n. 9, p. 2735- 2738, 2015.

LEE, Grace Young et al. Evaluation of a computer-assisted errorless learnig-based memory training program for patients with early Alzheimer's disease in Hong-Kong: a pilot study. Clinical Interventions in Aging, Auckland, v. 8, p. 623-633, 2013.

LETTS, Lori et al. Using occupations to improve quality of life, health and wellness and client and caregiver satisfaction for people with Alzheimer's disease and related dementias. The American Journal of Occupational Therapy, Bethesda, v. 65, n. 5, p. 497-504, set./out. 2011.

MANSUR, Letícia Lessa et al. Linguagem e cognição na doença de Alzheimer. Psicologia: reflexão e crítica, Porto Alegre, v. 18, n. 3, p. 300-307, set./dez. 2005.

MAPELLI, Daniela et al. Cognitive stimulation in patients with dementia: randomized controlled trial. Dementia and Geriatric Cognitive Disorders, Basiléia, v. 3, n. 1, p. 263-271, 2013. 
ORGANIZAÇÃO MUNDIAL DA SAÚDE (OMS). Classificação de transtornos mentais e de comportamento da CID-10: descrições clínicas e diretrizes diagnósticas. Porto Alegre: Artmed, 1993.

RADOMSKI, Mary Vining; DAVIS, Elin Schold. Otimização das capacidades cognitivas. In: RADOMSKI, Mary Vining; LATHAM, Catherine Trombly. Terapia Ocupacional para disfunções físicas. 6. ed. São Paulo: Santos, 2013. p. 749-773.

SCHABER, Patricia; LIEBERMAN, Deborah. Occupational Therapy Practice Guideline for Adults with Alzheimer's disease and related disorders. Bethesda: AOTA, 2010.

SERRANI AZCURRA, Daniel Jorge Luis. A reminiscence program intervention to improve the quality of life of long-term care residents with Alzheimer's disease: a randomized controlled trial. Revista Brasileira de Psiquiatria, Rio de Janeiro, v. 34, n. 4, p. 422-433, 2012.

SEYUN, Kim. Cognitive rehabilitation for elderly people with early-stage Alzheimer's disease. Journal of Physical Therapy Science, Tóquio, v. 27, n. 2, p. 543-546, 2015.

SOARES, Vera Lúcia Dias; SOARES, Cândida Dias; CAIXETA, Leonardo. Reabilitação neuropsicológica da memória. In: CAIXETA, Leonardo et al. Doença de Alzheimer. Porto Alegre: Artmed, 2012. p. 453-465.

SOHLBERG, McKay Moore; MATEER, Catherine Santo. Reabilitação cognitiva: uma abordagem neuropsicológica integrada. São Paulo: Santos, 2010.

THINNES, Andrea; PADILLA, René. Effect of educational and supportive strategies on the ability of caregivers of people with dementia to maintain participation in that role. The American Journal of Occupational Therapy, Bethesda, v. 65, n. 5, p. 541-549, set./out. 2011.

WERD, Maartje Me et al. Errorless learning of everyday tasks in people with dementia. Clinical Interventions in Aging, Bethesda, v. 8, n. 1, p. 1177-1190, 2013.

WOOD, Wendy; WOMACK, Jennifer; HOOPER, Barbara. Dying of boredom: an exploratory case study of time use, apparent affect, and routine activity situations on two Alzheimer's special care units. The American Journal of Occupational Therapy, Bethesda, v. 63, n. 3, p. 337-350, maio/jun. 2009.

WOODS, Bob et al. Cognitive stimulation to improve cognitive functioning in people with dementia. Cochrane Database of Systematic Reviews, San Diego, v. 15, n. 2, CD005562, 2012.

WORLD HEALTH ORGANIZATION (WHO). Dementia: a public health priority. Genebra: WHO, 2012

WU, Hua Shan et al. The effectiveness of spaced retrieval combined with Montessori-based activities in improving the eating ability of residents with dementia. Journal of Advanced Nursing, Medfore, v. 70, n. 8, p. 1891-1901, 2014.

YEN, Hsin-Yen; LIN, Li-Jung. A Systematic Review of Reminiscence Therapy for Older Adults in Taiwan. Journal of Nursing Research, v. 26, n. 2, p. 138-150, 2018.

YUILL, Natasha; HOLLIS, Vivien. A systematic review of cognitive stimulation therapy for older adults with mild to moderate dementia: an occupational therapy perspective. Occupational Therapy International, Montreal, v. 18, n. 4, p. 163-186, 2011.

Data de submissão: 20/07/2017

Data de aprovação: 05/03/2018 